\title{
No evidence for a causal link between uric acid and type 2 diabetes: a Mendelian randomisation approach
}

\author{
R. Pfister • D. Barnes $\cdot$ R. Luben • N. G. Forouhi • \\ M. Bochud • K.-T. Khaw • N. J. Wareham • \\ C. Langenberg
}

Received: 11 March 2011 /Accepted: 3 June 2011 / Published online: 30 June 2011

(C) Springer-Verlag 2011

\begin{abstract}
Aims/hypothesis Epidemiological and experimental evidence suggests that uric acid has a role in the aetiology of type 2 diabetes. Using a Mendelian randomisation approach, we investigated whether there is evidence for a causal role of serum uric acid for development of type 2 diabetes.

Methods We examined the associations of serum-uric-acidraising alleles of eight common variants recently identified in genome-wide association studies and summarised this in a genetic score with type 2 diabetes in case-control studies including 7,504 diabetes patients and 8,560 non-diabetic controls. We compared the observed effect size to that expected based on: (1) the association between the genetic
\end{abstract}

Electronic supplementary material The online version of this article (doi:10.1007/s00125-011-2235-0) contains supplementary material, which is available to authorised users.

R. Pfister · D. Barnes · N. G. Forouhi · N. J. Wareham •

C. Langenberg $(\bowtie)$

Medical Research Council Epidemiology Unit, Institute of Metabolic Science,

Box 285, Addenbrooke's Hospital, Hills Road,

Cambridge CB2 0QQ, UK

e-mail: claudia.langenberg@mrc-epid.cam.ac.uk

R. Pfister

Department III of Internal Medicine,

Heart Centre of the University of Cologne,

Cologne, Germany

R. Luben $\cdot$ K.-T. Khaw

Department of Public Health and Primary Care,

Institute of Public Health, University of Cambridge,

Cambridge, UK

M. Bochud

Institute of Social and Preventive Medicine,

Lausanne, Switzerland score and uric acid levels in non-diabetic controls; and (2) the meta-analysed uric acid level to diabetes association. Results The genetic score showed a linear association with uric acid levels, with a difference of $12.2 \mu \mathrm{mol} / 1$ (95\% CI $9.3,15.1)$ by score tertile. No significant associations were observed between the genetic score and potential confounders. No association was observed between the genetic score and type 2 diabetes with an OR of 0.99 (95\% CI 0.94, 1.04) per score tertile, significantly different $(p=0.046)$ from that expected (1.04 [95\% CI 1.03, 1.05]) based on the observed uric acid difference by score tertile and the uric acid to diabetes association of $1.21(95 \%$ CI $1.14,1.29)$ per $60 \mu \mathrm{mol} / \mathrm{l}$.

Conclusions/interpretation Our results do not support a causal role of serum uric acid for the development of type 2 diabetes and limit the expectation that uric-acid-lowering drugs will be effective in the prevention of type 2 diabetes.

Keywords Hyperuricaemia - Mendelian randomisation . Meta-analysis · Type 2 diabetes · Uric acid

$\begin{array}{ll}\text { Abbreviations } \\ \text { EPIC } & \begin{array}{l}\text { European Prospective Investigation into Cancer } \\ \text { and Nutrition }\end{array} \\ \text { GWAS } & \begin{array}{l}\text { Genome-wide association study } \\ \text { SNP }\end{array} \\ \text { Single nucleotide polymorphism }\end{array}$

Introduction

Observational studies have consistently reported significant positive associations between serum uric acid levels and incident diabetes, with a recent meta-analysis showing a $27 \%$ increase in risk per $60 \mu \mathrm{mol} / \mathrm{l}(1 \mathrm{mg} / \mathrm{dl})$ difference in 
uric acid levels [1]. However, serum uric acid levels are associated with many other diabetes risk factors and not all previous studies have adequately accounted for potential confounding factors. Furthermore, there is evidence for publication bias that may lead to an overestimation of the true association of serum uric acid levels on diabetes using published data [1]. Finally, hyperinsulinaemia can increase uric acid levels indicating that the direction of causality could be opposite, and raised serum uric acid levels could be a consequence rather than a cause of diabetes [2, 3]. Consequently, understanding the role of uric acid in diabetes requires further study, using methods that provide insights into the causal nature of observed associations.

Understanding whether serum uric acid levels are causally involved in the aetiology of type 2 diabetes has implications for public health and clinical practice. Both hyperuricaemia and diabetes are frequent in industrialised countries, and diabetes is known to be associated with an excess risk of morbidity and mortality [4]. If causal, hyperuricaemia will be a target for interventions for the prevention of type 2 diabetes. Assuming the effect estimate of the recent meta-analysis on serum uric acid levels and diabetes, treatment with allopurinol, the standard drug for lowering serum uric acid levels with an effect of about $180 \mu \mathrm{mol} / \mathrm{l}$ decrease in serum uric acid levels per $300 \mathrm{mg}$ allopurinol, would translate into a $51 \%$ risk reduction for diabetes [5]. This would be more effective than treatment with metformin and nearly as effective as multimodal lifestyle modifications in the high-risk individuals of the Diabetes Prevention Program [6]. Given the well-recognised possibility of residual confounding of the serum uric acid to diabetes association, it is important to quantify the unconfounded effect size of hyperuricaemia to estimate the true potential impact through intervention.

The concept of Mendelian randomisation is increasingly applied in epidemiology to test the causality of associations between risk factors and outcomes [7, 8]. Alleles are randomly allocated during gamete formation and thus the risk for confounding of the genetic variant to outcome association and for reverse causality is minimised. Accordingly, a genetic variant that is shown to be associated with the risk factor can be used to calculate an unconfounded estimate of the risk factor to outcome association and consequently can help to understand the causal nature of an observational association. This is true provided that certain assumptions are met [9].

In this study, we use a Mendelian randomisation approach to investigate whether we can find evidence for a causal link between serum uric acid levels and type 2 diabetes. We test the separate and joint effects of eight common genetic variants, recently identified as determinants of serum uric acid levels, on diabetes to compare the observed size of association to that expected based on: (1) the association between genetic variants and serum uric acid levels; and (2) the serum uric acid to diabetes association.

\section{Methods}

Our method was based on the triangulation approach described in Fig. 1. According to the concept of Mendelian randomisation, we used genetic variants as instrumental variables to disentangle the potentially confounded association between serum uric acid levels and type 2 diabetes. In summary, we used a genetic score based on eight common genetic variants previously shown to be associated with serum uric acid levels: (a) data from three type 2 diabetes case-control studies (Cambridgeshire, ADDITION-Ely and Norfolk Diabetes) to test the association between the genetic score and diabetes risk; (b) data from the European Prospective Investigation into Cancer and Nutrition (EPIC)Norfolk cohort and existing data from an additional seven cohorts to estimate the magnitude of the association between serum uric acid levels and diabetes; and (c) data from a subset of the EPIC-Norfolk cohort to estimate the association between the genetic score and serum uric acid levels to calculate (d) an approximate expected effect of the genetic score on diabetes assuming that serum uric acid levels are aetiologically associated with type 2 diabetes.

\section{Study populations}

Type 2 diabetes case-control studies Details of the three type 2 diabetes case-control studies, Cambridgeshire, ADDITION-Ely and Norfolk Diabetes, are reported in the electronic supplementary material [ESM].

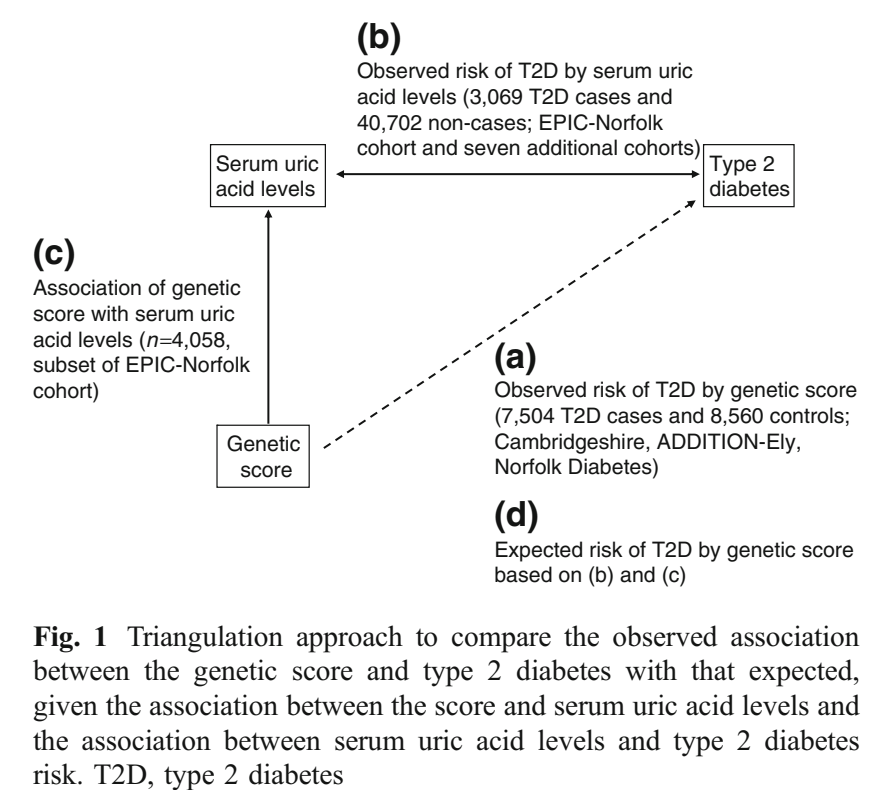

Observed risk of T2D by serum uric acid levels (3,069 T2D cases and 40,702 non-cases; EPIC-Norfolk

(d)

Expected risk of T2D by genetic score based on (b) and (c)

Fig. 1 Triangulation approach to compare the observed association between the genetic score and type 2 diabetes with that expected, given the association between the score and serum uric acid levels and risk. T2D, type 2 diabetes 
EPIC-Norfolk cohort study EPIC-Norfolk is a prospective cohort study in which men and women aged 40 to 79 years were recruited from general practices in the Norfolk region, UK. Full details of the population are reported elsewhere [10]. Between 1993 and 1997, 25,639 participants completed a health and lifestyle questionnaire, which asked about participants' personal and family health, demography, lifestyle, education and diet, and attended a clinic visit for health examination including assessment of non-fasting blood samples. Details of the assessment of baseline variables are described elsewhere [11-13]. A total of 787 verified incident cases of diabetes (mean [SD] age at diagnosis 68 years [8]) occurred before 31 July 2006, which were ascertained using several sources of evidence as previously described [14]. The EPIC-Norfolk Study was approved by the Norwich Local Research Ethics Committee.

We used the EPIC-Norfolk cohort to examine the association of serum uric acid levels with incident diabetes. Individuals with known cardiovascular disease, cancer or diabetes at baseline $(n=2,211)$ and individuals with missing covariates for multivariable analysis (age, sex, BMI, systolic blood pressure, cholesterol, HDL-cholesterol, triacylglycerol, WHR, educational level, smoking status, alcohol consumption or physical activity, $n=858$ ) were excluded. The final sample for this analysis was 15,496 participants in total and 449 participants with diabetes.

In addition, we used 6,455 participants randomly selected from the total EPIC-Norfolk cohort without known or incident diabetes and available baseline DNA samples as controls in the Norfolk Diabetes case-control study. These participants were also used to analyse the association between genetic variants/the genetic score on the one hand and serum uric acid levels and other diabetes risk factors (potential confounders) on the other; serum uric acid measures were available in 4,621 of these 6,455 participants as a result of the limited availability of stored baseline serum samples for analysis (see below).

Measurement of serum uric acid levels

Serum uric acid concentrations were measured in 2010 in all participants of the EPIC-Norfolk cohort with stored baseline serum samples available $(n=18,565)$ using a uricase/peroxidase method (Olympus AU640 clinical chemistry analyser, Olympus, Watford, UK). Analysis was performed according to the manufacturer's recommendation. The calibration range is 89 to $1,785 \mu \mathrm{mol} / 1$.

\section{Genotyping}

We genotyped single nucleotide polymorphisms (SNPs) recently identified as top hits in a genome-wide association study (GWAS) meta-analysis of serum uric acid levels [15] in and near PDZK1 (rs12129861), SLC2A9 (rs734553), ABCG2 (rs2231142), LRRC16A (rs742132), SLC17A1 (proxy rs1183201 for rs1165151, $r^{2}=1$ ), SLC16A9 (rs12356193), SLC22A11 (rs17300741) and SLC22A12 (rs505802) in our three case-control studies. Genotyping was performed with Custom TaqMan SNP Genotyping Assays (Applied Biosystems, Warrington, UK).

All eight variants passed quality control criteria separately in all studies (call rates $>95 \%$ and duplicate concordance $\geq 99 \%$ assessed in $1 \%$ of each study cohort). None of the SNPs showed substantial deviation from Hardy-Weinberg equilibrium ( $p$ values $\geq 0.01$ ) in non-diabetic controls. Allele frequencies were consistent with those reported for the CEU population (Centre d'Etude du Polymorphisme Humain [US residents with northern and western European ancestry]) of the HapMap (http://hapmap.ncbi.nlm.nih.gov/cgi-perl/ gbrowse/hapmap28_B36/).

\section{Statistical analyses}

Genotyping and genetic score We did not observe substantial deviation of the eight variants from an additive model on serum uric acid levels, and individual variants were coded as 0,1 and 2 on the basis of the number of the serum-uric-acid-increasing alleles. A genetic predisposition score was calculated for each individual by adding the number of serum-uric-acid-increasing alleles from all eight variants. In order to increase the effect size and thereby our statistical power, we divided the score into three equally sized groups ( 2 to 7,8 to 9 , and 10 to 15 risk alleles). As the effect size of the individual variants differs markedly, we also calculated a score by weighting the individual variants by the size of their effect on serum uric acid levels, using estimates from the recent GWAS meta-analysis. Given that the variant rs734553 in the $S L C 2 A 9$ gene has a very strong association with serum uric acid levels, results of this single variant are also presented.

Association between genetic variants and serum uric acid levels and potential confounders The association of individual genetic variants and the genetic score with serum uric acid levels was examined in a random subset of the EPIC-Norfolk cohort without diabetes using linear regression analysis adjusted for age and sex. One key assumption of Mendelian randomisation is that the genetic variants do not show pleiotropic effects, i.e. are not associated with other diabetes risk factors (potential confounders). To test this assumption we also examined the association of the genetic score with established risk factors of type 2 diabetes using linear regression analysis and the $\chi^{2}$ test. 
Association between serum uric acid levels and type 2 diabetes The relative risk of serum uric acid levels for incident diabetes was examined in participants of the EPICNorfolk cohort study using Cox proportional hazard regression with different degrees of adjustments. Consistent with earlier reports [16, 17], we observed a log-linear relation between serum uric acid levels and risk of diabetes (data not shown). We combined our result with five cohorts of European descent from a recent meta-analysis and a recent analysis of the Framingham study using a random effect model as we observed evidence for heterogeneity across studies $[1,18]$.

Association between genetic variants and type 2 diabetes Logistic regression analysis was used to calculate ORs for the association between individual variants and the genetic score with type 2 diabetes, assuming additive genetic models and adjusting for age, sex and BMI. ORs from each of the three case-control studies were combined by applying a fixed-effects meta-analysis model.

The expected association of the genetic variants on diabetes was calculated based on the meta-analysed association of serum uric acid levels on diabetes (OR 1.21 [95\% CI $1.14,1.29]$ per $60 \mu \mathrm{mol} / 1[1 \mathrm{mg} / \mathrm{dl}]$ serum uric acid) and the observed association of the genetic variants on serum uric acid levels: expected effect $=\exp$ (difference in serum uric acid levels $\left.\times \log _{e}[1.21]\right)$. This calculation takes into account the uncertainty in the estimation of the uric acid level to diabetes association, but not the uncertainty in the estimation of the genetic variant to uric acid levels association. The difference between observed and expected association was tested for statistical significance by an interaction test as described by Altman and Bland [19]. All analyses were performed using Stata version 10.1 (StataCorp, TX, USA).

\section{Results}

Association between genetic variants and both serum uric acid levels and potential confounders

Serum-uric-acid-raising alleles of all eight previously described variants were positively associated with serum uric acid levels in a random subset of the EPIC-Norfolk cohort without diabetes. The association with serum uric acid levels ranged from $2.2 \mu \mathrm{mol} / \mathrm{l}$ per risk allele for variant rs12129861 to $20.5 \mu \mathrm{mol} / 1$ per risk allele for variant rs734553 (Fig. 2), which is within the range described in a recent GWAS meta-analysis except for rs12129861, rs12356193, rs734553 and rs742132, which showed a slightly weaker association than previously described (difference 0.6$1.2 \mu \mathrm{mol} / \mathrm{l})$. Serum uric acid levels continuously increased with increasing tertiles of the genetic score (Fig. 3), with each tertile associated with an increase in serum uric acid levels of $12.2 \mu \mathrm{mol} / 1$ (95\% CI 9.3, 15.1, $p<0.0001$, adjusted for age and sex).

There was no evidence for a significant association between the genetic score and potential confounders of type 2 diabetes including BMI, blood pressure, total cholesterol, LDL- and HDL-cholesterol, triacylglycerol, smoking status, physical activity and alcohol consumption (ESM Table 1).

Association between serum uric acid levels and type 2 diabetes

Table 1 shows the association of serum uric acid levels with incident diabetes in the EPIC-Norfolk cohort as hazard ratios. There was a substantial attenuation of the association between serum uric acid levels and incident diabetes with increasing level of adjustment, with an HR of 1.47 per $60 \mu \mathrm{mol} / 1$ in the age- and sex-adjusted model compared
Fig. 2 Association of genetic variants with serum uric acid levels and type 2 diabetes. ${ }^{\text {a } E s t i m a t e s ~ r e s u l t ~ f r o m ~ a d d i t i v e ~}$ linear regression on serum uric acid levels (micromoles per litre) per one risk allele, adjusted for age and sex $(n=4,410-$ 4,571). ${ }^{\mathrm{b}}$ Estimates per one risk allele result from a fixed effect meta-analysis of the three casecontrol studies (maximum number of cases 7,504/controls $8,560)$, adjusted for age, sex and BMI. 'Proxy for rs1183201. T2D, type 2 diabetes; UA, uric acid

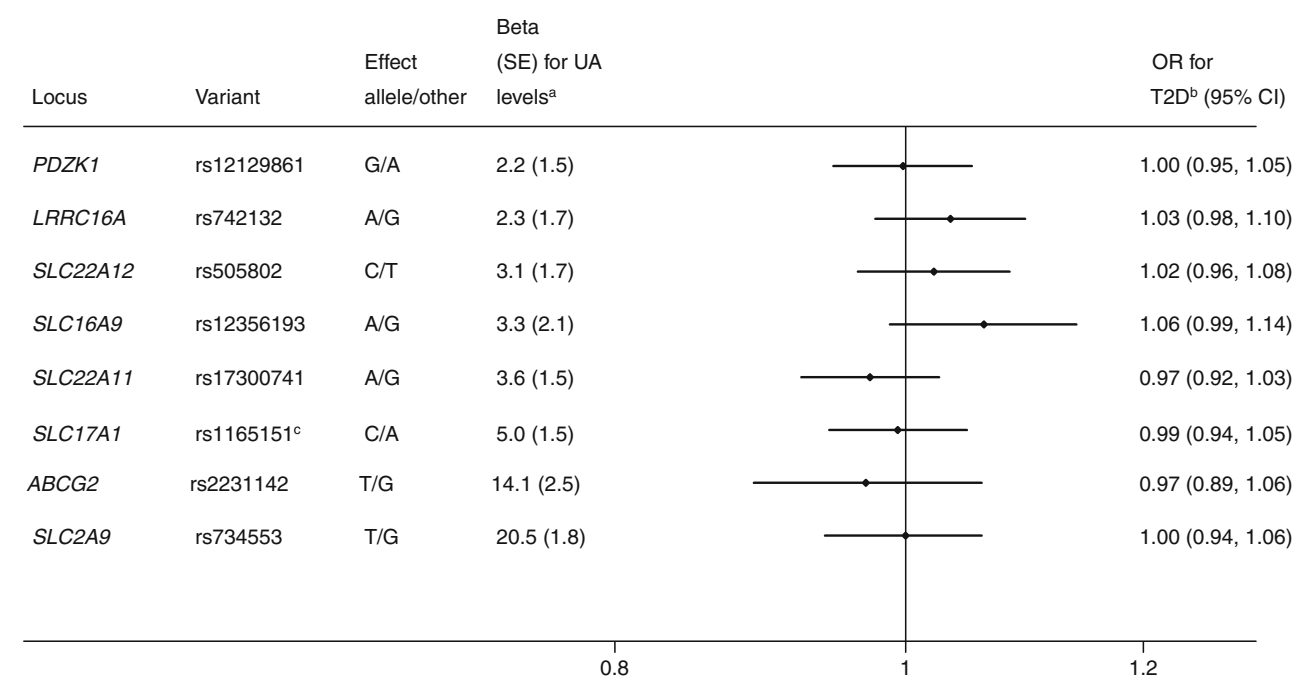




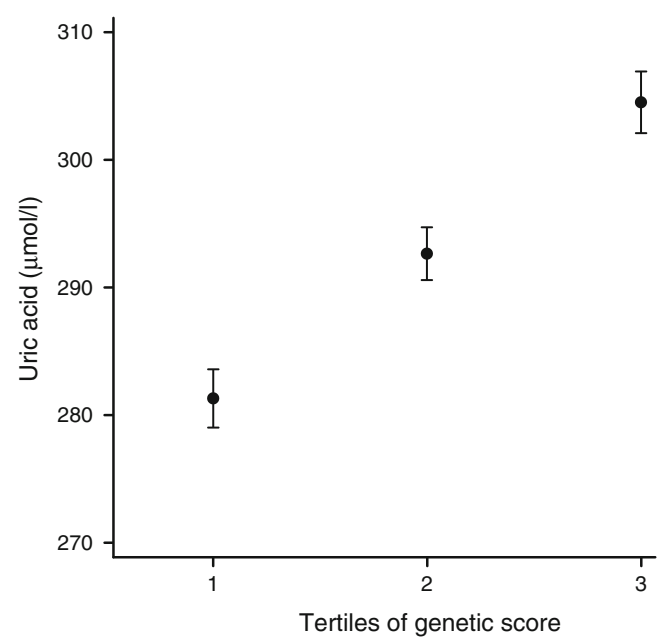

Fig. 3 Serum uric acid levels (mean \pm SE) by tertiles of the genetic risk score. Effective $n=4,058$ as a result of missing genotype information for one or more of the eight individual genetic variants of the score

with 1.18 per $60 \mu \mathrm{mol} / \mathrm{l}$ in the multiply adjusted model including age, sex, BMI, systolic blood pressure, cholesterol, HDL-cholesterol, triacylglycerol, WHR, educational level, smoking status, alcohol consumption and physical activity.

In a meta-analysis including our results and seven additional cohorts $[1,18]$ in total comprising 3,069 individuals with type 2 diabetes and 40,702 non-diabetic individuals, the relative risk of incident diabetes per $60 \mu \mathrm{mol} / 1$ difference in serum uric acid levels was 1.21 $(95 \%$ CI $1.14,1.29)$, with a range of RR of 1.04 to 1.63 for individual studies (Fig. 4) and evidence for significant heterogeneity across studies $\left(I^{2} 60.8 \%, p=0.005\right)$.

Association between genetic variants and type 2 diabetes

Pooled estimates from the meta-analysis of our three type 2 diabetes case-control studies comprising 7,504 cases and

Table 1 Multivariate adjusted relative risk of serum uric acid levels for incident type 2 diabetes in the EPIC-Norfolk cohort

\begin{tabular}{lll}
\hline Model & HR $(95 \% \mathrm{CI})^{\mathrm{a}}$ & $p$ value \\
\hline A & $1.47(1.37,1.57)$ & $<0.0001$ \\
B & $1.23(1.14,1.32)$ & $<0.0001$ \\
C & $1.18(1.10,1.28)$ & $<0.0001$ \\
\hline
\end{tabular}

${ }^{\mathrm{a}} \mathrm{HR}$ is per $60 \mu \mathrm{mol} / \mathrm{l}(1 \mathrm{mg} / \mathrm{dl})$ increase in uric acid levels, analysis included 449 diabetes cases in a total sample of 15,496

A: adjusted for age and sex, B: adjusted as model A plus BMI, C: adjusted as model B plus systolic blood pressure, cholesterol, HDLcholesterol, triacylglycerol, WHR, educational level, smoking status, alcohol consumption, physical activity
8,560 controls showed no significant association between the genetic score and diabetes, with an OR of $0.99(95 \% \mathrm{CI}$ $0.94,1.04, p=0.62$, Fig. 5) per genetic score tertile. There was no evidence for a significant association between the genetic score and diabetes within the three individual casecontrol studies $(p>0.32)$. The pooled estimates of the individual genetic variants varied between 0.97 and 1.06 per risk allele, with an OR of 1.00 (95\% CI 0.94, 1.06) for the variant rs734553 (Fig. 2). There was no evidence of heterogeneity between the three case-control studies ( $I^{2}$ between $0 \%$ and $43.1 \%, p$ values $>0.19$ ).

The expected association between the genetic score and type 2 diabetes (according to Fig. 1d) based on the genetic score to serum uric acid level association $(12.2 \mu \mathrm{mol} / \mathrm{l}$ per tertile $[95 \%$ CI $9.3,15.1])$ and the meta-analysed serum uric acid level to diabetes association (OR 1.21 [95\% CI $1.14,1.29]$ per $60 \mu \mathrm{mol} / 1[1 \mathrm{mg} / \mathrm{dl}]$ serum uric acid) was $1.04(95 \%$ CI $1.03,1.05)$. This is significantly different from that observed $(z=2.00, p=0.046$, Fig. 5) suggesting that a genetically determined increase in serum uric acid levels is not associated with increased risk of diabetes. The difference between expected and observed association with type 2 diabetes was also significant for the variant rs 734553 $(z=1.98, p=0.048)$.

When analysing the weighted genetic score, the expected association between score and type 2 diabetes (OR 1.05 [95\% CI $1.04,1.07]$ per tertile) was also significantly different from that observed (OR 0.99 [95\% CI 0.95, 1.05]). The difference between expected and observed association was more pronounced for the weighted score $(z=2.19, p=$ 0.029) compared with the unweighted score, which was due to a stronger effect of the weighted score on serum uric acid levels (difference of $16.4 \mu \mathrm{mol} / \mathrm{l}$ in serum uric acid levels per tertile of the weighted score, ESM Fig. 1).

\section{Discussion}

\section{Main findings}

Our epidemiological analysis in a large cohort of European descent confirmed previous data showing a significant association between serum uric acid levels and development of type 2 diabetes after multivariable adjustment. However, we found substantial evidence for confounding. Using an approach based on the concept of Mendelian randomisation, we found no support for a causal role of serum uric acid levels in the development of type 2 diabetes. We observed a linear increase in serum uric acid levels with increasing number of risk alleles summarised to a genetic predisposition score, but there was no association between this score and the risk for diabetes. 
Fig. 4 Meta-analysis of the relative risk of serum uric acid levels for type 2 diabetes. RR, with corresponding $95 \% \mathrm{CIs}$, refers to a $60 \mu \mathrm{mol} / 1[1 \mathrm{mg} / \mathrm{dl}]$ increase in serum uric acid levels. The area of each square is proportional to study weight. Diamond indicates overall RR; horizontal lines indicate $95 \%$ CIs. ${ }^{a}$ Data from Kodama et al. [1]
Study ID

$\operatorname{RR}(95 \% \mathrm{Cl})$

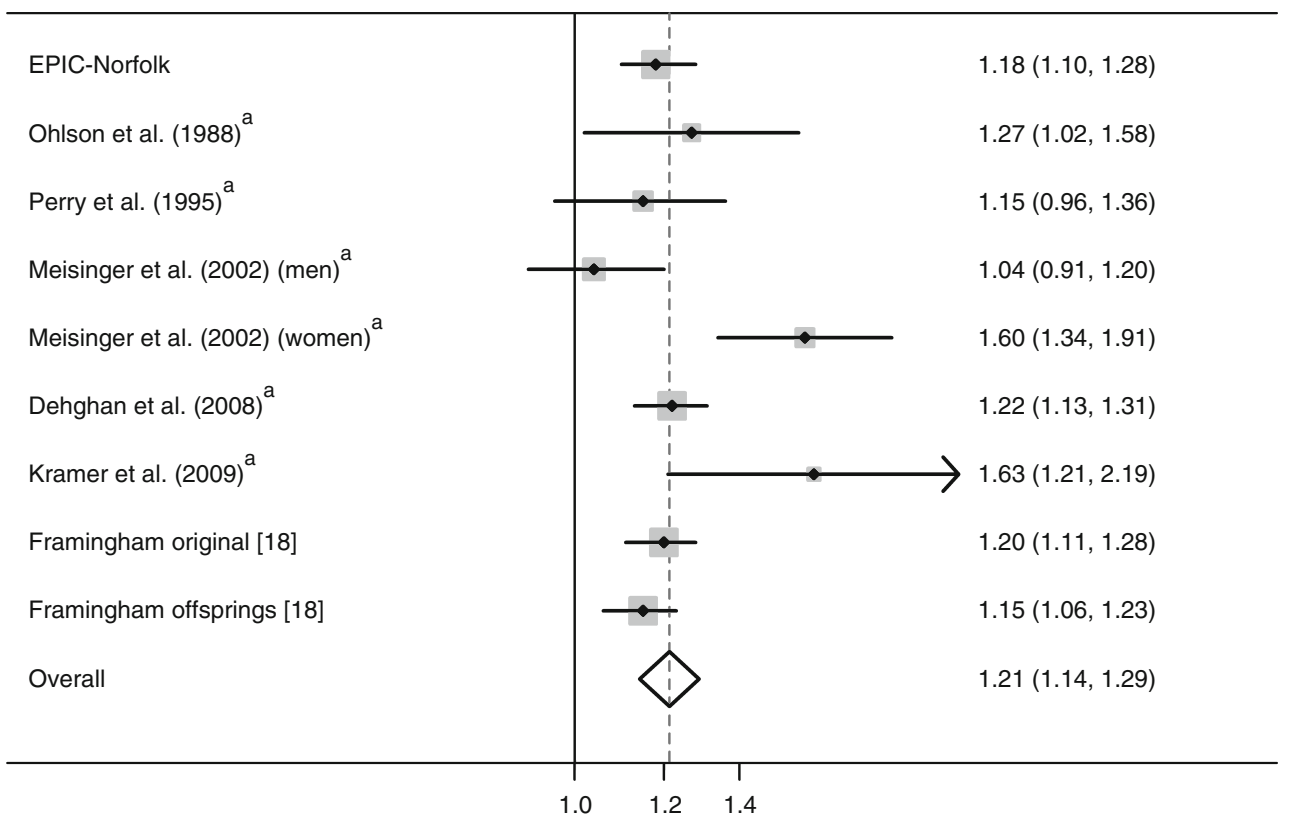

Is serum uric acid causally related to type 2 diabetes?

So far there is a body of evidence supporting a causal role of serum uric acid for development of type 2 diabetes, fulfilling most of the traditional criteria for causality in epidemiology. The association of uric acid levels and incident diabetes is strong, consistent and linear. We confirmed this in a meta-analysis comprising 3,069 incident cases of diabetes and 40,702 non-cases. Elevated serum levels of uric acid often precede hyperinsulinaemia and diabetes [17, 20]. Lowering of serum uric acid levels can prevent metabolic-syndrome-like phenotypes in animals [21]. Hyperuricaemia can induce endothelial dysfunction and oxidative stress, both known mechanisms contributing to the development of the metabolic syndrome and type 2 diabetes [22, 23]. Mechanistically, fructose (e.g. in the form of sweetened beverages), which is strongly linked to obesity, can increase serum uric acid levels, which could potentially mediate an association with an adverse metabolic phenotype [24]. However, there is also evidence for residual confounding, publication bias and reverse causality, resulting in a possible overestimation of the true association. To our knowledge, there is no large randomised controlled trial of serum-uric-acid-lowering interventions in humans that could contribute evidence for or against a causal role of uric acid in the development of type 2 diabetes. Recent randomised controlled trials examining the effects of allopurinol did not report a significant effect on glucometabolic traits
Fig. 5 Comparison of observed and expected association of the genetic score and variant rs 734553 with type 2 diabetes. ${ }^{\mathrm{a}}$ Observed vs expected effect: $p<0.05$
Variant

Effect

Odds ratio $(95 \% \mathrm{Cl})$

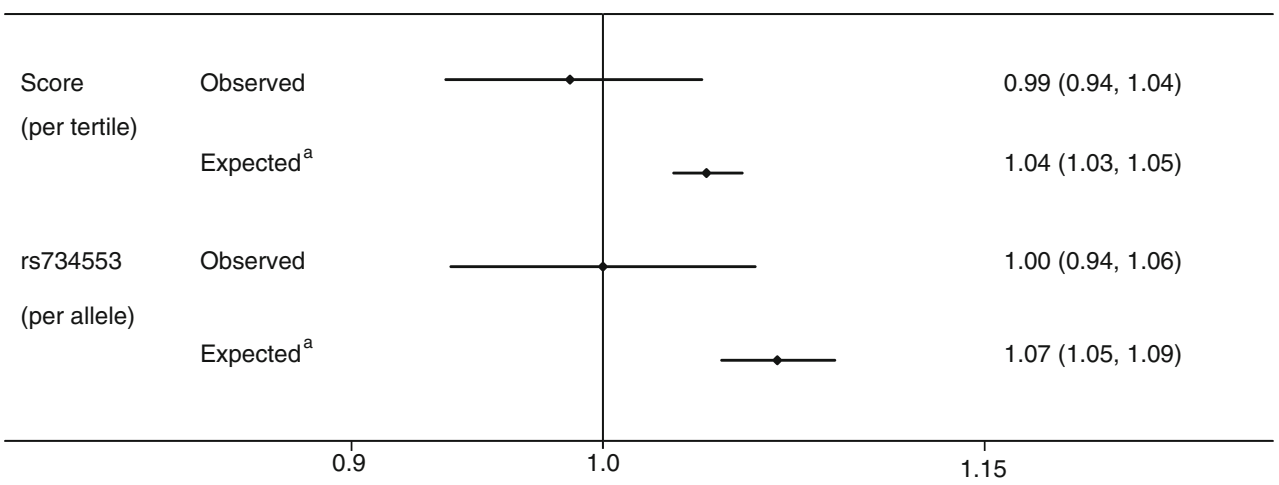


$[25,26]$. However, the implication of these studies is limited given the small samples $(n<75)$, short follow-up ( $<1$ month) and pleiotropic effects of allopurinol. One of the uses of the Mendelian randomisation approach is in prioritising pathways in which clinical trials might be justified, which is necessary given the high cost of such trials. The evidence from our Mendelian randomisation analysis of uric acid levels suggests that the investment in a large uric-acidlowering intervention trial in people at risk of diabetes would not be justified.

Our findings add to existing genetic evidence against a causal role of uric acid in metabolic and cardiovascular disease. The genetic variant in the SLC2A9 locus was not associated with the metabolic syndrome or hypertension in large case-control studies [27-29]. Further, none of the established serum uric acid variants was associated with coronary heart disease in two recent studies, although these studies had low statistical power [30, 31].

We suggest that serum uric acid is a secondary phenomenon of an adverse metabolic phenotype and residual confounding may be responsible for the association with type 2 diabetes found in observational studies. Of note, only a few studies corrected for alcohol consumption and smoking and hardly any studies corrected for renal function or use of diuretics, which are associated with diabetes and serum uric acid levels [32, 33]. Further, physical activity may also confound the association and adequate correction is limited by imperfect measurement [34]. We demonstrated a substantial attenuation of the association between serum uric acid levels and incident diabetes after extensive adjustment. The relative risk difference per $60 \mu \mathrm{mol} / 1[1 \mathrm{mg} / \mathrm{dl}]$ increase in serum uric acid levels fell from $47 \%$ to $18 \%$ after adjustment.

\section{Clinical implications}

Current recommendations on drug treatment of hyperuricaemia are based on the presence of complications that are shown to be causally related to serum uric acid, such as gout or urolithiasis [5]. Our results do not support a causal role of serum uric acid for type 2 diabetes and hence do not encourage the initiation of clinical trials or an expansion of serum-uric-acid-lowering interventions with the aim of preventing type 2 diabetes. This might be of major relevance as allopurinol is one of the drugs most frequently involved in adverse reactions following inappropriate prescription [35], which might in part be due to confusion by the ongoing discussion on a causal role of serum uric acid for type 2 diabetes and other metabolic disease [36]. However, some of the non-pharmacological interventions recommended for the lowering of serum uric acid levels, such as weight loss, diet modifications, restriction of alcohol consumption and physical exercise, have positive effects beyond the lowering of serum uric acid and are clearly appropriate in metabolic high-risk individuals [37].

Strengths and limitations

Strengths and limitations of our study are closely linked to the applied method of Mendelian randomisation. The advantage of this method is that it allows a more definite conclusion on causality compared with traditional observational studies [7]. Nevertheless, we acknowledge the limitation of trying to estimate the average causal effect for binary outcomes [9]. There are a few conditions that have to be followed. First, there has to be a robust and strong association between genetic variant and risk factor [7]. The eight variants examined here were described in a meta-analysis of GWAS on serum uric acid levels including more than 28,000 individuals. Seven of the eight variants were replicated in independent population-based studies including 7,795 to 11,024 participants, with similar size of association as in the GWAS meta-analysis $[38,39]$. We observed similar associations as in the GWAS meta-analysis in four of the eight variants and slightly weaker associations in the remaining four variants, suggesting that we had maybe underestimated the gene to serum uric acid level association, and the difference between expected and observed association would be even larger.

The second condition is that the genetic variants are not related to typical confounders, which was tested and confirmed in an extensive analysis within one of the control cohorts. Third, the observed association between genetic variants and outcome is only mediated by the risk factor of interest, i.e. the variants show no pleiotropy or direct effects on outcome. This assumption relies on background knowledge of the underlying biology, which is never complete, and thus cannot be proven in practice. However, we consider that, given current knowledge on the biology of these genes, this is a reasonable assumption. A priori we did not include the variant rs780094 within the 'glucokinase regulatory protein' locus in our analysis although it was significantly associated with serum uric acid levels in GWAS meta-analysis. The variant rs780094 was shown to be associated with pancreatic beta cell function and several metabolic traits such as, for example, fasting glucose, cholesterol and triacylglycerol levels, with the latter being independent of uric acid levels, thus strongly suggesting a violation of key assumptions of Mendelian randomisation by this variant $[39,40]$.

Importantly, seven of our eight variants are close to or within genes responsible for cellular transport of uric acid and are therefore strong functional candidates influencing serum uric acid levels. This knowledge on the biological function of the variants might reduce the risk of pleiotropy. Nonetheless, we cannot exclude the possibility that our 
variants are in linkage disequilibrium with variants that influence type 2 diabetes by other pathways.

The application of a score consisting of several genetic markers may lead to a violation of the crucial assumption of Mendelian randomisation of the random assortment of variants. However, linkage disequilibrium between each of the eight markers is small (all $r^{2}<0.1$ ) and only three pairs of variants are located on the same chromosome. Furthermore, our case-control populations are entirely of European ancestry, which limits the generalisability of our results. Moreover, our estimates are based on a single measurement of serum uric acid levels. The variation of serum uric acid levels over time in healthy individuals was shown to be low, however, with a coefficient of variation of $9 \%$ [41]. Hence, bias by regression-dilution effect might be limited. Finally, our sample size of 7,504 cases and 8,560 controls does not provide sufficient statistical power to detect small effects of the genetic variants on type 2 diabetes.

In summary, our results provide no support for a causal role for serum uric acid for the development of type 2 diabetes and suggest that it is unlikely that serum-uric-acidlowering drugs would be effective in the prevention of type 2 diabetes.

Acknowledgements We thank all study participants, the study team, S. Griffin, MRC Epidemiology Unit, for assistance with the ADDITION study and the technical teams of the MRC Epidemiology Unit and the EPIC-Norfolk Study group for genotyping and informatics support. Cambridgeshire, Ely, ADDITION, EPIC-Norfolk and the Norfolk Diabetes studies were funded by the MRC with support from NHS Research \& Development and the Wellcome Trust. R. P. received grants from Koeln Fortune and Marga- und Walter-Boll Stiftung. The funders had no role in study design, data collection and analysis, decision to publish or preparation of the manuscript.

R.P. was responsible for study design, analyses, manuscript draft, D.B. for statistical analysis and contribution to final manuscript version, R.L. for data management, statistical analysis and contribution to final manuscript version, N.G.F. for study design and contribution to final manuscript version, M.B. for analyses and contribution to the final manuscript version, K.-T.K. for study design and contribution to the final manuscript version, N.W. for study design and contribution to final manuscript version, and C.L. for study design and manuscript draft. All authors approved the final version of the manuscript to be published.

Duality of interest The authors declare that there is no duality of interest associated with this manuscript.

\section{References}

1. Kodama S, Saito K, Yachi Y et al (2009) Association between serum uric acid and development of type 2 diabetes. Diabetes Care 32:1737-1742

2. Quinones GA, Natali A, Baldi S et al (1995) Effect of insulin on uric acid excretion in humans. Am J Physiol 268:E1-E5

3. Fox IH (1981) Metabolic basis for disorders of purine nucleotide degradation. Metabolism 30:616-634
4. Haffner SM, Lehto S, Ronnemaa T, Pyorala K, Laakso M (1998) Mortality from coronary heart disease in subjects with type 2 diabetes and in nondiabetic subjects with and without prior myocardial infarction. N Engl J Med 339:229-234

5. Zhang W, Doherty M, Bardin T et al (2006) EULAR evidence based recommendations for gout. Part II: Management. Report of a task force of the EULAR Standing Committee for International Clinical Studies Including Therapeutics (ESCISIT). Ann Rheum Dis 65:1312-1324

6. Knowler WC, Barrett-Connor E, Fowler SE et al (2002) Reduction in the incidence of type 2 diabetes with lifestyle intervention or metformin. N Engl J Med 346:393-403

7. Sheehan NA, Didelez V, Burton PR, Tobin MD (2008) Mendelian randomisation and causal inference in observational epidemiology. PLoS Med 5:e177

8. Smith GD, Ebrahim S (2003) "Mendelian randomization": can genetic epidemiology contribute to understanding enviromental determinants of disease? Int J Epidemiol 32:1-22

9. Didelez V, Sheehan N (2007) Mendelian randomization as an instrumental variable approach to causal inference. Stat Methods Med Res 16:309-330

10. Day N, Oakes S, Luben R et al (1999) EPIC-Norfolk: study design and characteristics of the cohort. European Prospective Investigation of Cancer. Br J Cancer 80(Suppl 1):95-103

11. Khaw KT, Wareham N, Bingham S, Luben R, Welch A, Day N (2004) Association of hemoglobin A1c with cardiovascular disease and mortality in adults: the European prospective investigation into cancer in Norfolk. Ann Intern Med 141:413420

12. McFadden E, Luben $\mathrm{R}$, Wareham N, Bingham S, Khaw KT (2008) Occupational social class, educational level, smoking and body mass index, and cause-specific mortality in men and women: a prospective study in the European Prospective Investigation of Cancer and Nutrition in Norfolk (EPIC-Norfolk) cohort. Eur J Epidemiol 23:511-522

13. Wareham NJ, Jakes RW, Rennie KL et al (2003) Validity and repeatability of a simple index derived from the short physical activity questionnaire used in the European Prospective Investigation into Cancer and Nutrition (EPIC) Study. Public Health Nutr 6:407-413

14. Patel PS, Sharp SJ, Luben RN et al (2009) Association between type of dietary fish and seafood intake and the risk of incident type 2 diabetes: the European prospective investigation of cancer (EPIC)-Norfolk cohort study. Diabetes Care 32:1857-1863

15. Kolz M, Johnson T, Sanna S et al (2009) Meta-analysis of 28,141 individuals identifies common variants within five new loci that influence uric acid concentrations. PLoS Genet 5:e1000504

16. Chien KL, Chen MF, Hsu HC et al (2008) Plasma uric acid and the risk of type 2 diabetes in a Chinese community. Clin Chem $54: 310-316$

17. Dehghan A, van Hoek M, Sijbrands EJ, Hofman A, Witteman JC (2008) High serum uric acid as a novel risk factor for type 2 diabetes. Diabetes Care 31:361-362

18. Bhole V, Choi JW, Kim SW, de Vera M, Choi H (2010) Serum uric acid levels and the risk of type 2 diabetes: a prospective study. Am J Med 123:957-961

19. Altman DG, Bland JM (2003) Interaction revisited: the difference between two estimates. BMJ 326:219

20. Nakanishi N, Okamoto M, Yoshida H, Matsuo Y, Suzuki K, Tatara K (2003) Serum uric acid and risk for development of hypertension and impaired fasting glucose or type II diabetes in Japanese male office workers. Eur J Epidemiol 18:523-530

21. Nakagawa T, Hu H, Zharikov S et al (2006) A causal role for uric acid in fructose-induced metabolic syndrome. Am J Physiol Renal Physiol 290:F625-F631 
22. Khosla UM, Zharikov S, Finch JL et al (2005) Hyperuricemia induces endothelial dysfunction. Kidney Int 67:1739-1742

23. Sautin YY, Nakagawa T, Zharikov S, Johnson RJ (2007) Adverse effects of the classic antioxidant uric acid in adipocytes: NADPH oxidase-mediated oxidative/nitrosative stress. Am J Physiol Cell Physiol 293:C584-C596

24. Johnson RJ, Perez-Pozo SE, Sautin YY et al (2009) Hypothesis: could excessive fructose intake and uric acid cause type 2 diabetes? Endocr Rev 30:96-116

25. Perez-Pozo SE, Schold J, Nakagawa T, Sanchez-Lozada LG, Johnson RJ, Lillo JL (2010) Excessive fructose intake induces the features of metabolic syndrome in healthy adult men: role of uric acid in the hypertensive response. Int J Obes (Lond) 34:454-461

26. George J, Carr E, Davies J, Belch JJ, Struthers A (2006) High-dose allopurinol improves endothelial function by profoundly reducing vascular oxidative stress and not by lowering uric acid. Circulation 114:2508-2516

27. Caulfield MJ, Munroe PB, O’Neill D et al (2008) SLC2A9 is a high-capacity urate transporter in humans. PLoS Med 5:e197

28. Vitart V, Rudan I, Hayward C et al (2008) SLC2A9 is a newly identified urate transporter influencing serum urate concentration, urate excretion and gout. Nat Genet 40:437-442

29. McKeigue PM, Campbell H, Wild S et al (2010) Bayesian methods for instrumental variable analysis with genetic instruments ('Mendelian randomization'): example with urate transporter SLC2A9 as an instrumental variable for effect of urate levels on metabolic syndrome. Int J Epidemiol 39:907-918

30. Stark K, Reinhard W, Grassl M et al (2009) Common polymorphisms influencing serum uric acid levels contribute to susceptibility to gout, but not to coronary artery disease. PLoS One 4:e7729

31. Yang Q, Kottgen A, Dehghan A et al (2010) Multiple genetic loci influence serum urate levels and their relationship with gout and cardiovascular disease risk factors. Circ Cardiovasc Genet 3:523530
32. Lin CS, Hung YJ, Chen GY et al (2011) A multicenter study of the association of serum uric acid, serum creatinine, and diuretic use in hypertensive patients. Int JCardiol 148:325-330

33. Villegas R, Xiang YB, Cai Q et al (2010) Prevalence and determinants of hyperuricemia in middle-aged, urban Chinese men. Metab Syndr Relat Disord 8:263-270

34. Wareham NJ, Rennie KL (1998) The assessment of physical activity in individuals and populations: why try to be more precise about how physical activity is assessed? Int J Obes Relat Metab Disord 22(Suppl 2):S30-S38

35. Jonville-Bera AP, Saissi H, Bensouda-Grimaldi L et al (2009) Avoidability of adverse drug reactions spontaneously reported to a French regional drug monitoring centre. Drug Saf 32:429440

36. Suzuki I, Yamauchi T, Onuma M, Nozaki S (2009) Allopurinol, an inhibitor of uric acid synthesis - can it be used for the treatment of metabolic syndrome and related disorders? Drugs Today (Barc) 45:363-378

37. Jordan KM, Cameron JS, Snaith M et al (2007) British Society for Rheumatology and British Health Professionals in Rheumatology guideline for the management of gout. Rheumatology (Oxford) 46:1372-1374

38. Dehghan A, Kottgen A, Yang Q et al (2008) Association of three genetic loci with uric acid concentration and risk of gout: a genome-wide association study. Lancet 372:1953-1961

39. van der Harst P, Bakker SJ, de Boer RA et al (2010) Replication of the five novel loci for uric acid concentrations and potential mediating mechanisms. Hum Mol Genet 19:387-395

40. Bi M, Kao WH, Boerwinkle $\mathrm{E}$ et al (2010) Association of rs780094 in GCKR with metabolic traits and incident diabetes and cardiovascular disease: the ARIC Study. PLoS One 5:e11690

41. Yu KH, Luo SF, Tsai WP, Huang YY (2004) Intermittent elevation of serum urate and 24-hour urinary uric acid excretion. Rheumatology (Oxford) 43:1541-1545 